NASA TECHNICAL MEMORANDUM

$\frac{8}{7}$
$\frac{8}{5}$
$\frac{5}{2}$

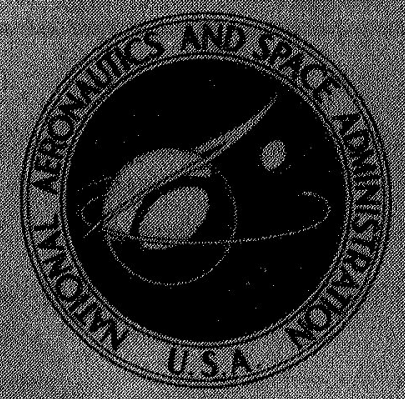

NASA TM X-1680

GPO PRICE

CFSTI PRICE(S) \$

Hard copy $(\mathrm{HC})$

Microfiche (MF) -

ff 653 July 65

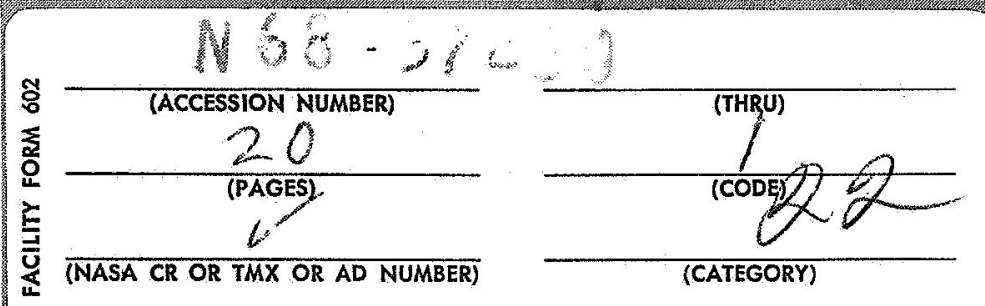

GAMMA HEATING IN THIN

HEAVY-ELEMENT ABSORBERS

by John H. Lyuch, Richard J. Crum, and Harry J. Reilly

Lewis Research Center

Cleveland, Obio

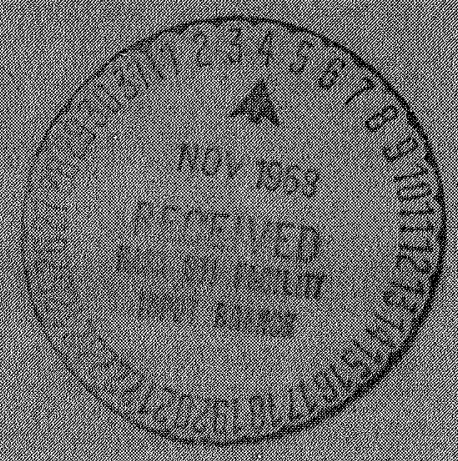

NATIONAL AERONAUTICS AND SPACE ADMINISTRATION - WASHINGTON, D. C. - OCTOBER 1968 
NASA TM X-1680

\section{GAMMA HEATING IN THIN HEAVY-ELEMENT ABSORBERS}

By John H. Lynch, Richard J. Crum, and Harry J. Reilly

Lewis Research Center

Cleveland, Ohio

NATIONAL AERONAUTICS AND SPACE ADMINISTRATION

For sale by the Clearinghouse for Federal Scientific and Technical Information

Springfield, Virginja 22151 - CFSTI price $\$ 3.00$ 


\section{ABSTRACT}

The ratio of gamma heating per gram of tungsten to gamma heating per gram of water was calculated for the case of a thin tungsten detector in a water shield. One-dimensional transport theory calculations were used to obtain response polynomials which predict this ratio as a function of source energy, shield thickness, and detector thickness. Electron transport effects were also examined. Useful results are presented in the form of graphs. 


\title{
GAMMA HEATING IN THIN HEAVY-ELEMENT ABSORBERS
}

\author{
by John H. Lynch, Richard J. Crum, and Harry J. Reilly \\ Lewis Research Center
}

\begin{abstract}
SUMMARY
Two effects must be considered when converting the gamma heating rate measured using an aqueous dosimeter to the gamma heating rate in a thin tungsten detector immersed in a water shield. These are, first, the difference in heating due to the difference between the mass energy absorption coefficients of the heavy detector and the aqueous dosimeter, and second, the "skin heating effect. "1 The measured heating rates must be corrected to account for these effects. Calculated thin detector heating rates which are obtained using the buildup factor method also require correction for these effects because the buildup factor does not take into account any physical properties of the detector.

One-dimensional photon transport-theory calculations were performed to obtain correction factors for these effects for tungsten detectors in a water shield. The results of these calculations were used to construct polynomial equations which predict the combined magnitude of the effects as a function of gamma source energy (from 0.255 to 4. $0 \mathrm{MeV}$ ), water-shield thickness (from 4.0 to $44.0 \mathrm{~cm}$ ), and tungsten-detector thickness (from 0.00254 to $0.762 \mathrm{~cm}$ ). Polynomials were also obtained for the gamma heating $(\mathrm{W} / \mathrm{g})$ in water and in tungsten so that multienergy sources can be analyzed.

The behavior of the correction factor ( $\mathrm{W} / \mathrm{g}$ in tungsten divided by $\mathrm{W} / \mathrm{g}$ in water) around the center point (2.128-MeV source, $24.0-\mathrm{cm}$ water shield, and $0.044-\mathrm{cm}$ detector) was examined using these polynomials. This ratio was also investigated for a fission spectrum source. The ratio was less than two for detector thickness greater than 0.2 centimeter. The polynomials fit the data with an average absolute error of less than 10.2 percent.

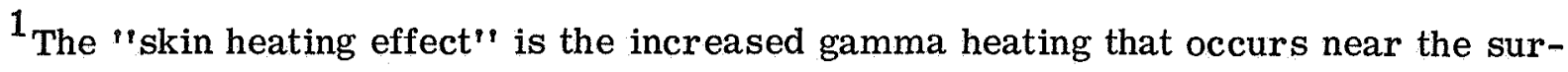
face of the detector. This heating results from the influx of a large quantity of lowenergy Compton scattered gammas. These gammas are absorbed locally due to the large photoelectric cross sections of the heavy element detector.
\end{abstract}




\section{INTRODUCTION}

Reactor irradiation experiments may have substantial internal heating by gamma rays from the core and surrounding structures. Often it is important to measure the amount of gamma heat either for heat-transfer design calculations or to determine effects on temperature measurements.

Gamma heating at a given location in water or other light elements can be determined using conventional aqueous detectors such as ferrous sulfate dosimeters. However, such detectors, although convenient to use, do not give gamma energy spectral information which is needed to compute what the heating would be at the same location using a heavy element such as tungsten.

Therefore a conventional way to do design calculations involving heavy elements is first to measure the gamma heating using an aqueous dosimeter, obtaining watts per gram in water. This watts per gram is multiplied by the mass of an experiment component to obtain the heat generation in that component. The result is then multiplied by a correction factor for the mass energy absorption coefficient and skin-heating effects.

There has been a lack of good information on these correction factors. The problem has been identified in the literature (refs. 1 to 3), but the choice of the correction factors has remained mostly a matter of engineering judgment. It is the purpose of this report to show accurate calculated values for these correction factors for the most general case of a point source and a shell detector. Also shown are the calculational model and the synthetic experiment design techniques that were used to select the calculations that were performed. The results are presented in the form of polynomial response equations. Special cases of interest are shown, and applications of the results are discussed at the conclusion of the report.

\section{SYMBOLS}

$\begin{array}{ll}\mathrm{A}_{\mathrm{o}}, \mathrm{A}_{\mathrm{n}}, \mathrm{A}_{\mathrm{nn}} & \text { constant term or coefficient in a response equation } \\ \mathbf{E} & \text { energy, } \mathrm{MeV} \\ \mathrm{E}_{\mathrm{O}} & \text { uncoded source energy, } \mathrm{MeV} \\ \mathbf{F} & \text { gamma heating correction factor from response equation } \\ \mathrm{F}^{\prime} & \text { computed gamma heating correction factor from transport calculations } \\ \mathbf{f} & \text { fraction of electron energy lost while escaping } \\ \mathbf{P} & \text { escape probability for electrons } \\ \mathbf{R} & \text { extrapolated range of electrons in tungsten, } \mathrm{cm}\end{array}$


$\mathbf{r}$ radius coordinate in transport model, $\mathrm{cm}$

S multienergy source, photons $/(\mathrm{sec})(\mathrm{MeV})$

T untransformed, uncoded tungsten-detector thickness, $\mathrm{cm}$

t untransformed, uncoded water-shield thickness, cm

t* transformed, uncoded water-shield thickness

$\mathrm{X}_{1} \quad$ coded source energy

$\mathrm{X}_{2} \quad$ transformed and coded water-shield thickness

$\mathrm{X}_{3}$ transformed and coded tungsten-detector thickness

$\gamma \quad$ relative gamma heating, $\mathrm{W} / \mathrm{g}$

$\theta \quad$ plane angle corresponding to $\Omega$

$\psi \quad$ fraction of electron kinetic energy that is deposited in detector

$\Omega \quad$ solid angle

Subscripts:

$\mathrm{n}, \mathrm{nn} \quad$ variable indicators in response equation

1 number of experiment

Superscripts:

W tungsten

$\mathrm{H}_{2} \mathrm{O}$ water

\section{METHOD OF ANALYSIS}

The model selected was a point source of gammas immersed in a spherical water shield. The detector consisted of a spherical tungsten shell. A water backstop outside of the detector was used with a vacuum boundary condition (see fig. 1). Fluxes were obtained using DTF-IV, a discrete ordinates computer solution to the Boltzmann equations (ref. 4). Scattering cross sections were generated using GAMLEG (ref. 5). Gamma absorption cross sections were taken from reference 6. Energy absorption coefficients were obtained using data from reference 7 corrected to include energy deposited by Compton scattering. To guarantee reliable answers, between 13 and 23 energy groups with $\mathrm{S}_{12}$ Gaussian angular quadrature were used with the $\mathrm{P}_{3}$ scattering approximation.

The correction factor $F$ was defined as the ratio of the gamma heating in the tungsten detector to the gamma heating in a water detector having the same thickness and location (see eqs. (1) and (2)). This ratio was obtained from 15 sets of two companion 


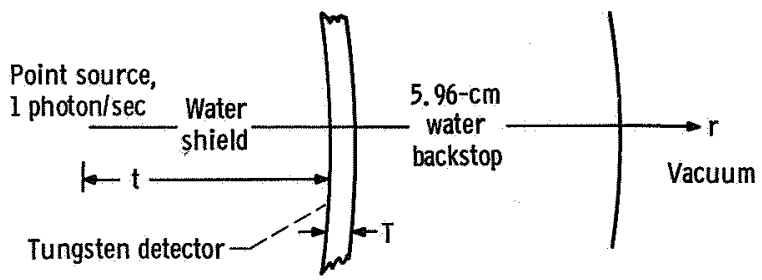

Figure 1. - Geometrical model used in gamma transport calculations.

calculations which were the same in all respects except that one calculation of each set had a tungsten detector and the other had a water detector.

To obtain the maximum information possible from a limited amount of calculations, the correction factor was studied using multivariate regression analysis. This analysis used the calculations as synthetic experiments in an orthogonal composite design (ref. 8). This approach produced a response polynomial that may be used to compute the correction factor for any values of the independent variables over the range of the variables studied. The independent variables selected were source energy, shield thickness (water thickness between the source and the tungsten), and detector thickness. A summary of the experiment statistical design is given in appendix $\mathrm{A}$.

Because the expected range of correction factors was from 1 to about 30, a least squares fit based on minimizing the sum of the squares of the residuals would make the fit poor for the smaller values. For this reason the natural logarithm of the correction factor was fit instead of fitting the correction factor directly. This fit minimizes the sum of squares of the fractional errors at the observed points if the errors are not large (see appendix A).

The orthogonal composite design guarantees that the effects of independent variables and their interactions are truly separated (i. e., their correlation coefficients are zero). This gives the most accurate response equation. However, because the levels at which two the independent variables must be selected for orthogonality are undesirably spaced, new variables were defined which improved the fits. These were obtained by using cubic or logarithmic transformations of the originally selected base variables of shield thickness and detector thickness. These transformations are discussed in appendix A. Source energy was not transformed.

\section{RESULTS}

The correction factor $\mathbf{F}$ for monoenergetic source gammas is defined as 


$$
F\left(t, T, E_{0}\right) \equiv \frac{\int_{0}^{E_{0}} \int_{t}^{t+T} \gamma^{W}(t, r, E) r^{2} d r d E}{\int_{0}^{E_{0}} \int_{t}^{t+T} \gamma^{H_{2}{ }^{O}}(t, r, E) r^{2} d r d E}
$$

For multienergy sources, the appropriate $F$ factor is defined by

$$
F(t, T) \equiv \frac{\int_{0}^{\infty} \int_{t}^{t+T} S(E) \gamma^{W}(t, r, E) r^{2} d r d E}{\int_{0}^{\infty} \int_{t}^{t+T} S(E) \gamma^{H_{2} O}{ }_{(t, r, E) r^{2} d r d E}}
$$

where

$\mathrm{S}(\mathrm{E}) \quad$ multienergy source, photons/(sec)(MeV)

$\gamma^{\mathrm{W}}(\mathrm{t}, \mathrm{r}, \mathrm{E}) \quad$ gamma heating in tungsten, $\mathrm{W} / \mathrm{g}$

$\gamma^{\mathrm{H}_{2} \mathrm{O}}(\mathrm{t}, \mathrm{r}, \mathrm{E})$ gamma heating in water, $\mathrm{W} / \mathrm{g}$

The ranges of variables studied were $0.00254-$ to 0.762 -centimeter detector thickness $(\mathrm{T}), 0.255-$ to $4.0-\mathrm{MeV}$ source energy $\left(\mathrm{E}_{\mathrm{o}}\right)$, and 4.0 - to 44.0 -centimeter water-shield thickness (t).

Polynomials were generated for $F\left(t, T, E_{0}\right), \gamma^{W}\left(t, T, E_{0}\right)$, and $\gamma^{\mathrm{H}_{2} \mathrm{O}}\left(\mathrm{t}, \mathrm{T}, \mathrm{E}_{\mathrm{o}}\right)$. The form of these polynomials is

In $\mathrm{K}=\mathrm{A}_{\mathrm{o}}+\mathrm{A}_{1} \mathrm{X}_{1}+\mathrm{A}_{2} \mathrm{X}_{2}+\mathrm{A}_{3} \mathrm{X}_{3}+\mathrm{A}_{11} \mathrm{X}_{1}^{2}+\mathrm{A}_{12} \mathrm{X}_{1} \mathrm{X}_{2}+\mathrm{A}_{13} \mathrm{X}_{1} \mathrm{X}_{3}+\mathrm{A}_{22} \mathrm{X}_{2}^{2}$

$$
+A_{23} X_{2} X_{3}+A_{33} X_{3}^{2}
$$

where $\mathrm{x}_{1}, \mathrm{x}_{2}$, and $\mathrm{x}_{3}$ are coded and transformed values of the source energy, watershield thickness, and tungsten-detector thickness, and $\mathrm{K}$ is $\mathrm{F}, \gamma^{\mathrm{W}}$, or $\gamma^{\mathrm{H}_{2} \mathrm{O}}$. These independent variables are defined by equations (A2) to (A4) in appendix A. The polynomial coefficients are shown in table $I$. 
TABLE I. - POLYNOMIAL COEFFICIENTS FOR EQUATION (3)

\begin{tabular}{|c|c|c|c|}
\hline Coefficient & $\mathrm{K}=\mathrm{F}\left(\mathrm{t}, \mathrm{T}, \mathrm{E}_{\mathrm{o}}\right)$ & $\mathrm{K}=\gamma^{\mathrm{W}}\left(\mathrm{t}, \mathrm{T}, \mathrm{E}_{\mathrm{o}}\right)$ & $\mathrm{K}=\gamma^{\mathrm{H}_{2} \mathrm{O}}\left(\mathrm{t}, \mathrm{T}, \mathrm{E}_{\mathrm{o}}\right)$ \\
\hline $\mathrm{A}_{0}$ & 0.8301 & 1.5922 & 0.7640 \\
$\mathrm{~A}_{1}$ & -.6221 & .2488 & .8697 \\
$\mathrm{~A}_{2}$ & .1829 & -.1784 & -.3605 \\
$\mathrm{~A}_{3}$ & -.8018 & 1.5433 & 2.3412 \\
$\mathrm{~A}_{11}$ & .5137 & .0743 & -.4421 \\
$\mathrm{~A}_{12}$ & -.0084 & .1101 & .1205 \\
$\mathrm{~A}_{13}$ & .4169 & .4173 & -.0015 \\
$\mathrm{~A}_{22}$ & -.1447 & -.1342 & .0119 \\
$\mathrm{~A}_{23}$ & -.1614 & -.1653 & .0003 \\
$\mathrm{~A}_{33}$ & .0181 & .0770 & .0558 \\
\hline
\end{tabular}

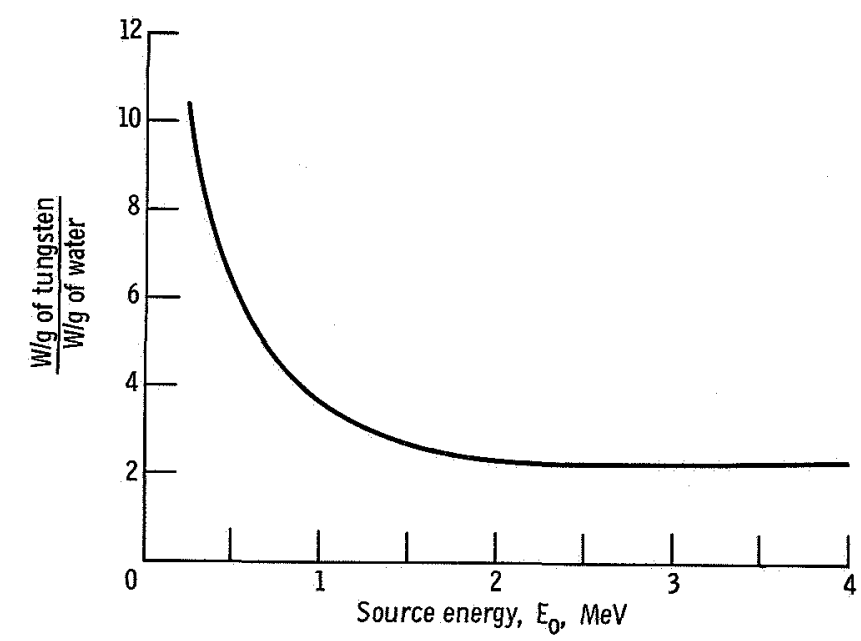

Figure 2. - Gamma heating correction factor $F$ for a 24-centimeter water shield and a 0.044 -centimeter tungsten detector as function of source energy.

In order to acquire some feel for the behavior of $F\left(t, T, E_{0}\right)$ when the gamma source is monoenergetic, equation (3) was evaluated over the complete range of each independent variable around the design center (figs. 2 to 4 ). These figures may be used when the independent variables are approximately those used to draw the curves. However, because of the interaction terms, it is advisable to use equation (3) if an accurate $F$ factor is desired. The polynomials are valid only over the range of variables studied. Hence, extrapolation, especially for thinner detectors, is not recommended.

The polynomials in $\gamma^{\mathrm{W}}$ and $\gamma^{\mathrm{H}_{2} \mathrm{O}}$ can be used to evaluate $\mathrm{F}$ for a multienergy source (eq. (2)). Equation (2) was approximated for several water-shield and detector 


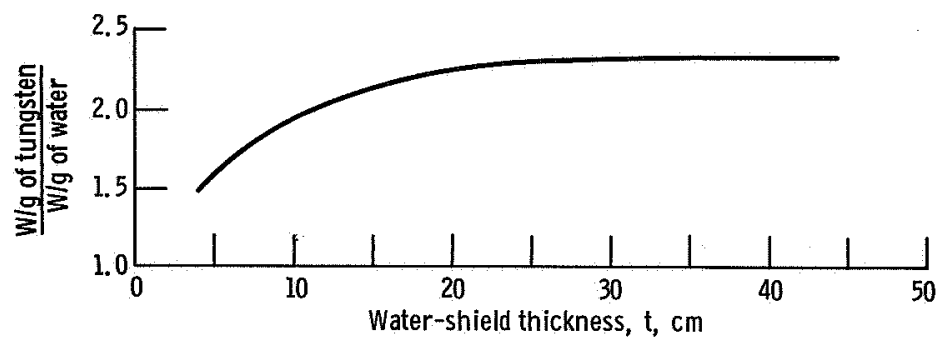

Figure 3. - Gamma heating correction factor $\mathrm{F}$ for 2. 128-MeV gammas and a 0.044 -centimeter tungsten detector as function of shield thickness.

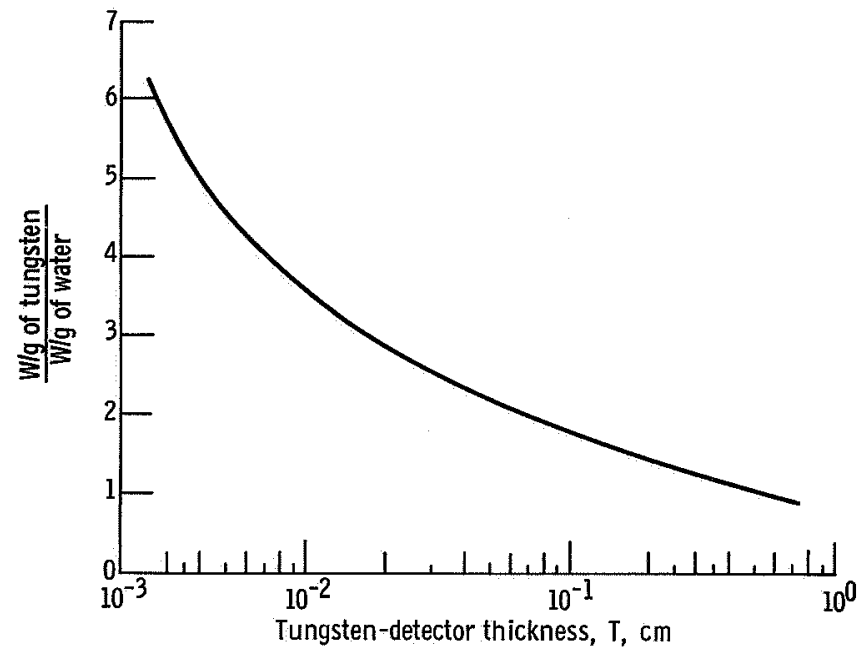

Figure 4. - Gamma heating correction factor $F$ for 2. 128-MeV gammas and a 24-centimeter water shield as function of tungsten-detector thickness.

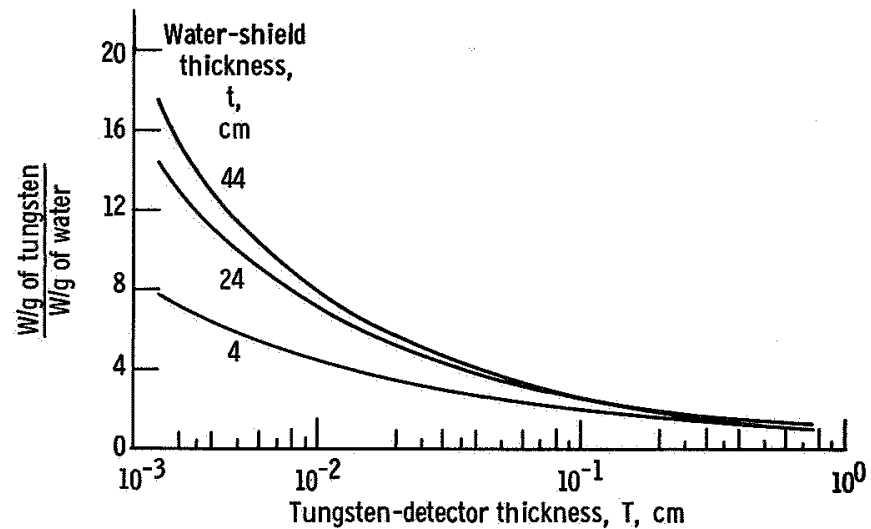

Figure 5. - Gamma heating correction factor $F$ for fission spectrum gammas as function of tungsten-detector thickness $T$ with water-shield thickness $t$ as a parameter. 
thicknesses by using a five-energy group representation of the Carver prompt plus delayed fission gamma spectrum (ref. 9). These results are shown in figure 5.

\section{ERROR ANALYSIS}

\section{Uncertainty in the Polynomial Fits}

Table II is a comparison of the calculated $\mathrm{F}, \gamma^{\mathrm{W}}$, and $\gamma^{\mathrm{H}_{2} \mathrm{O}}$ values obtained from the DTF calculations and the polynomial approximations of these values. The percent error at each point and statistical measures of accuracy are also given. The errors at the experimental points provide a first-order estimate of the error that can be expected for intermediate points calculated using the polynomials (see experiments 16 and 17 shown in table II). The statistical measures of fit tend to verify the selection of variables and to provide some indication of the overall error and dispersion in the fits.

If a more exact estimate of the uncertainty is desired, the 95-percent confidence interval associated with equation (3) may be computed using

$$
\begin{aligned}
& \text { Confidence interval }=\ln F \pm 2.57 \sigma \\
& \quad(\text { for } \ln F)
\end{aligned}
$$

where

$$
\begin{aligned}
\sigma=0.2158\left[0.0667+0.0913\left(\mathrm{x}_{1}^{2}+\mathrm{x}_{2}^{2}+\mathrm{x}_{3}^{2}\right)+0.2291\left(\mathrm{x}_{1}^{4}+\mathrm{x}_{2}^{4}+\mathrm{x}_{3}^{4}\right)\right. & \\
& \left.+0.1250\left(\mathrm{x}_{1}^{2} \mathrm{x}_{2}^{2}+\mathrm{x}_{1}^{2} \mathrm{x}_{3}^{2}+\mathrm{x}_{2}^{2} \mathrm{x}_{3}^{2}\right)\right]^{1 / 2}
\end{aligned}
$$

and 2.57 is the two-sided student $t$ for 95-percent confidence with 5 degrees of freedom. This means that the true value of $\ln F$ will lie in an interval whose bounds are $\pm 2.57 \sigma$ from the polynomial approximation to $\ln \mathrm{F}$. The constants in this equation were taken from the inverted sum-of-cross-products-deviations matrix (ref. 10). Confidence intervals about $F$ for multienergy sources are complicated by the uncertainty in the integration resulting from the energy group scheme selected. This uncertainty was not examined. 

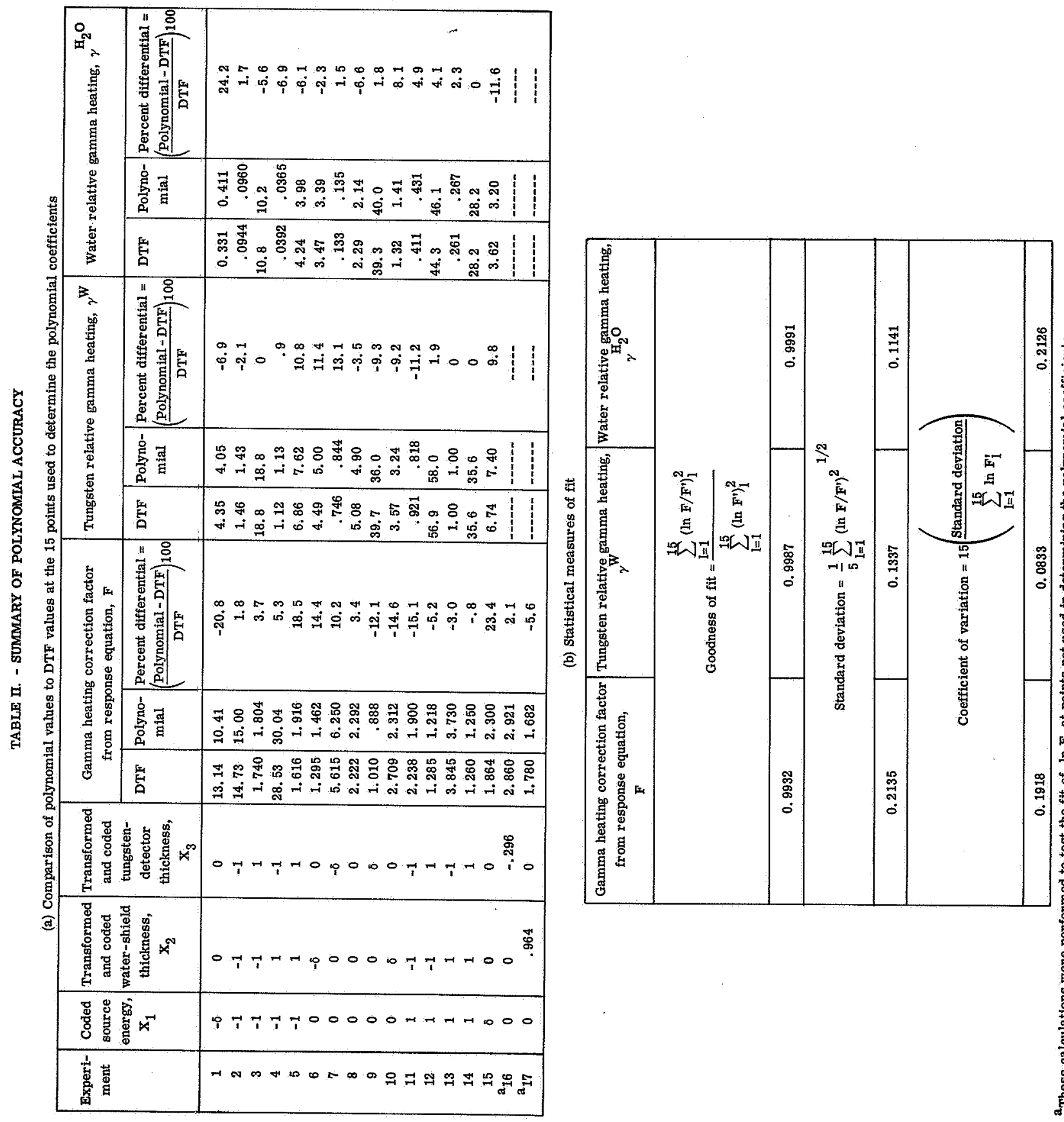


\section{Use of Numerical Model}

Hopefully some of the inaccuracy due to the numerical model cancels when the results of separate solutions are divided to obtain F. There is no general way to assess the accuracy of an iterative finite difference model. However, because of the large number of energy groups, $P_{3}$ scattering, fine mesh, and tight convergence used, the errors due to numerical procedure should be small.

\section{Errors in Cross Sections}

A detailed discussion of estimated cross section errors is given in reference 6 . In general, the errors can approach 10 percent below $50 \mathrm{KeV}$ but probably do not exceed 3 to 5 percent above $100 \mathrm{KeV}$.

\section{Electron Transport Effects}

The $\mathbf{F}$ values obtained refer to total heat deposition. For the thinnest detectors, however, part of the heat is deposited outside of the detector due to transport and escape of the electrons. A first-order model shown in appendix B was used to construct a function $\psi$, which is defined as the fraction of the electron kinetic energy that is retained in the tungsten detector as a function of initial electron energy. In the unperturbed calculations (without the tungsten), the net electron transport is negligible. The $\psi$ function is shown in figure 6.

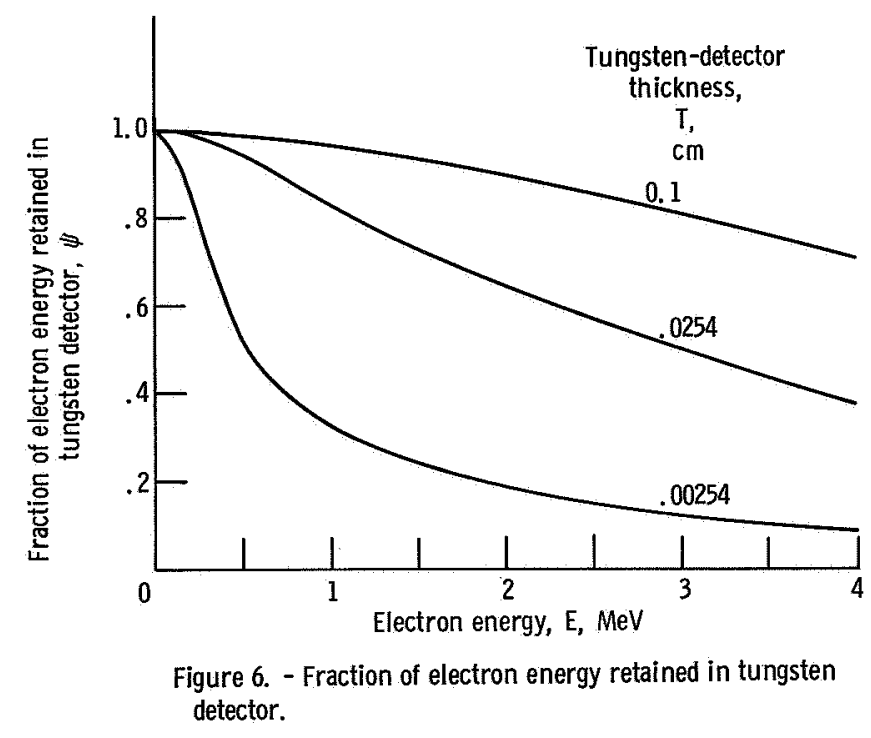


The correction factor $F$ becomes large only when most of the gamma flux incident on the detector is below $1.0 \mathrm{MeV}$ when the photoelectric effect dominates the gamma heating (e. g. , from fig. 3 for a 2.128-MeV gamma source, F becomes appreciable with a few centimeters of water shield thickness). Figure 6 shows that for reactions below $1 \mathrm{MeV}$ and thicknesses as small as 0.025 centimeter, the correction for electron transport is small. For thinner detectors the $F$ values should be corrected using the model shown in appendix $B$ or a similar treatment.

\section{USES OF THESE RESULTS}

The polynomials presented here provide a convenient method for computation of heating rates in tungsten using measurements made in water. The results have the quoted accuracy only for tungsten. However, it is reasonable to assume that other materials such as steel, lead, and uranium can be investigated using the same technique. In fact, it is suggested that the results given here could be used with reasonable success for other combinations of light element shields and heavy element detectors, given that they are treated in terms of some "equivalent thicknesses" of water detector and tungsten shield.

Polynomials such as these could also possibly be used in the place of conventional buildup factors in line-of-sight calculations for thin detectors. That is, they provide a buildup factor that is a function of the detector in addition to being a function of $\mu$ t of the shield. It should not be difficult to add such a feature to some of the widely used shielding codes of this type such as QADHD (ref. 11).

The problem considered here has been examined as a gamma problem without specifying the type of nuclear reaction that is required to produce the gammas. When these results are used to correct gamma heating measurements made in a reactor environment, consideration should be given to the fact that secondary gammas from neutron captures in the water shield and tungsten detector were not included in this model. Calculation of the heating due to secondary gammas requires knowledge of the neutron flux and must be treated as a separate problem. When the conversion factors given here are used with point kernel integration codes, they can be applied directly.

As has been noted, the results given here are conservative for very thin detectors in that electron losses from these detectors will carry some of the energy away from the detector to adjacent structures. The model shown for electron leakage should be useful for estimating this effect. Even with corrections for electron transport, however, the polynomials should not be used for absolute values of a variable greater than 1.2154.

Lew is Research Center,

National Aeronautics and Space Administration, Cleveland, Ohio, June 26, 1968, 120-27-04-54-22. 


\section{APPENDIX A}

\section{SUMMARY OF EXPERIMENT DESIGN}

To generate the response polynomials, 15 pairs of transport calculations were performed. Each pair was treated as an "experiment." The experimental statistical design used was an orthogonal composite fractional factorial. Coded levels of independent variables for each of the experiments are shown in figure 7.

Maximum and minimum values were selected for each of the chosen independent variables, and the intermediate values were linearly interpolated and coded as shown in reference 6. The values used in the DTF calculations are given in table III.

In order to distribute the calculational information more uniformly over the range of

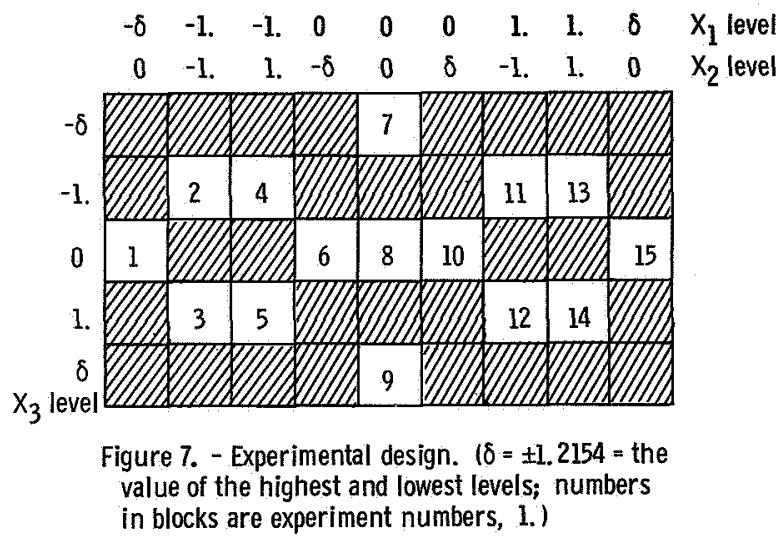

TABLE III. - VALUES OF INDEPENDENT

VARIABLES USED IN DTF

CALCULATIONS

\begin{tabular}{|c|c|c|c|}
\hline Level & $\begin{array}{c}\text { Source energy, } \\
E_{0 \prime} \\
\mathrm{MeV}\end{array}$ & $\begin{array}{c}\text { Water-shield } \\
\text { thickness, } \\
t^{\mathrm{a}} \\
\mathrm{cm}\end{array}$ & $\begin{array}{c}\text { Detector } \\
\text { thickness, } \\
\mathrm{T},{ }^{\mathrm{a}} \\
\mathrm{cm}\end{array}$ \\
\hline$-\delta$ & 0.255 & 4.00 & 0.00254 \\
-1 & .587 & 12.85 & .00421 \\
0 & 2.128 & 24.00 & .0440 \\
1 & 3.670 & 35.15 & .460 \\
$\delta$ & 4.000 & 44.00 & .762 \\
\hline
\end{tabular}

$a_{\text {Require transformation to be orthogonal. }}$ 
water-shield thickness selected for study, the water thicknesses shown in table III were used in the DTF calculations. To maintain the orthogonality of the experiment design, the water-shield thickness $t$ was transformed to the new variable $t *$ using

$$
t^{*}=\left(\frac{t-24}{10}\right)^{1 / 3}
$$

Also, since the detector thickness effects were anticipated to be largest for the thinnest detector, the level spacing dictated by the orthogonal design did not provide information in the region of highest importance. For this reason, the log of the detector thickness was used as the third variable rather than the dector thickness.

The equations that define the coded and transformed $\mathrm{X}$ values required for evaluation of the polynomials are

$$
\begin{aligned}
\mathrm{X}_{1} & \equiv \frac{\mathrm{E}_{\mathrm{o}}-2.128}{1.541} \\
\mathrm{X}_{2} & \equiv\left(\frac{\mathrm{t}-24.0}{11.15}\right)^{1 / 3} \\
\mathrm{X}_{3} & \equiv \frac{\ln \mathrm{T}+3.124}{2.347}
\end{aligned}
$$

Coded versions of the polynomials have been used here because the resulting response is not as sensitive to errors in independent variables as would be the response from polynomials using uncoded input.

The least squares fitting procedure minimizes the sum of squares of residuals at observed points. For the gamma heating correction factor, for example, it is desirable to obtain a fit which is as reliable for low values as it is for high ones. To accomplish this, the fit was made to $\ln F$ instead of $F$. The quantity that was minimized was then

$$
\sum_{l=1}^{15}\left[\ln F^{\prime}-\ln F\right]_{l}^{2}=\sum_{l=1}^{15}\left[\ln \frac{F^{\prime}}{F}\right]_{l}^{2}=\sum_{l=1}^{15}\left[\ln \frac{F+d F}{F}\right]_{l}^{2}
$$

The primes in equation (A5) denote observed values of $F$. If the log is expanded for a small argument (assuming that $\mathrm{dF}$, the error in $\mathrm{F}$, is small), equation (A5) becomes 


$$
\sum_{\mathrm{l}=1}^{15}\left(\frac{\mathrm{dF}}{\mathrm{F}}\right)_{1}^{2} \approx \text { function minimized by least squares }
$$

This gave a much better fit to the data than the fit of $F$ directly because fractional errors were being minimized. This reasoning was also used with the polynomials for $\gamma^{\mathrm{W}}$ and $\gamma^{\mathrm{H}_{2} \mathrm{O}}$. 


\section{APPENDIX B}

\section{DERIVATION OF $\psi$}

A function $\psi$ was derived which showed, to first order, the fraction of the calculated gamma heating that is retained in the tungsten detector. The $\psi$ function is defined as

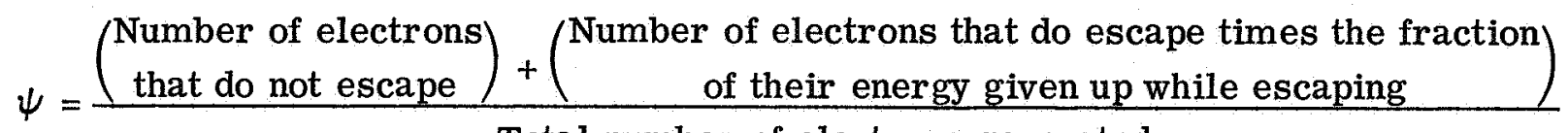

Total number of electrons generated

$$
=1-P(1-f)
$$

where

P electron escape probability

f fraction of electron energy that is lost during escape

To obtain P, two cases must be considered. First, when $R$, the extrapolated range of the electrons (ref. 12), is greater than or equal to $T$, the detector thickness, the escape probability is given by

$$
\mathrm{P}=\frac{\int_{0}^{\mathrm{T}}\left[1-\frac{1}{2}\left(\cos \theta_{1}+\cos \theta_{2}\right)\right] \mathrm{dx}}{\int_{0}^{\mathrm{T}} \mathrm{dx}}=1-\frac{\mathrm{T}}{2 \mathrm{R}}
$$

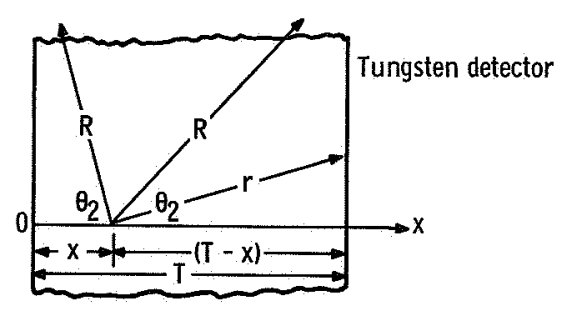

Figure 8. - Slab model used to obtain $P$ when $R \geq T$.

(The sum of the solid angles, obtained by rotating

$R$ about the $X$ axis, divided by $4 \pi$ gives the prob-

ability of escape for an electron born at $X$. This is

integrated over $X$ and divided by $T$ to obtain $P$

when $R \geq T$, ) 
where the slab model of figure 8 has been used to define $\theta_{1}$ and $\theta_{2}$. These angles are. the plane angles corresponding to the solid angles obtained by rotating the range vector $\mathbf{R}$ about the $\mathrm{X}$ axis. When $\mathrm{R} \leq \mathrm{T}$, a similar integration gives

$$
\mathbf{P}=\frac{\mathbf{R}}{2 \mathrm{~T}}
$$

To obtain $f$, the average distance $\bar{r}$ through the tungsten travelled by an escaping electron is first computed. This is used with the range energy relation (ref. 12) to compute the energy required (energy lost) to travel this distance. The fraction of energy lost during escape is this energy divided by the initial energy of the electron. The initial energy is assumed to be that of the photon causing the reaction.

When $\mathrm{R} \geq \mathrm{T}$,

$$
\overline{\mathrm{r}}=\frac{\int_{0}^{\mathrm{T}} \int_{0}^{\Omega} \mathrm{c} \mathrm{r} d \Omega \mathrm{dx}}{\int_{0}^{\mathrm{T}} \int_{0}^{\Omega} \mathrm{c} d \Omega d x}=\frac{\mathrm{T}\left(\ln \frac{\mathrm{R}}{\mathrm{T}}+\frac{1}{2}\right)}{2\left(1-\frac{\mathrm{T}}{2 \mathrm{R}}\right)}
$$

where use has again been made of the slab model shown in figure 8 and $\Omega_{c}$ is the solid angle corresponding to $\mathrm{r}=\mathrm{R}$.

When $\mathbf{R} \leq \mathrm{T}$, a similar integration gives

$$
\overline{\mathrm{r}}=\frac{\mathrm{R}}{2}
$$

Figure 6 was constructed using equations (B2) to (B5) in equation (B1). 


\section{REFERENCES}

1. Fox, Raymond: Gamma and Neutron Heating in and About a Homogeneous Reactor. Nucl. Sci. Eng., vol. 6, no. 1, July 1959, pp. 33-36.

2. Bopp, C. D.; and Towns, R. L.: Calorimetric Measurement of Nuclear Heating in a Reactor. Nucl. Sci. Eng., vol. 13, no. 3, July 1962, pp. 245-249.

3. Unruh, W. G.; and Tomlinson, M.: Mean Gamma-Ray Energy Absorption Coefficients. Nucl. Appl., vol. 3, Sept. 1967, pp. 548-549.

4. Lathrop, K. D. : DTF-IV - A FORTRAN-IV Program for Solving the Multigroup Transport Equation with Anisotropic Scattering. Rep. LA-3373, Los Alamos Scientific Lab., July 15, 1965.

5. Lathrop, K. D. : GAMLEG - A FORTRAN Code to Produce Multigroup Cross Sections for Photon Transport Calculations. Rep. LA-3267, Los Alamos Scientific Lab., Mar. 11, 1965.

6. Grodstein, Gladys W. : X-Ray Attenuation Coefficients from $10 \mathrm{KeV}$ to $100 \mathrm{MeV}$. Circ. 583, National Bureau of Standards, Apr. 30, 1957.

7. Hubbell, J. H.; and Berger, M. J.: Photon Attenuation and Energy Absorption Coefficients Tabulations and Discussion. Second ed., Rep. 8681, National Bureau of Standards, Sept. 28, 1966.

8. Krase, J. M.; and Cyl-Champlin, C.: Synthetic Experiment Design Techniques in Reactor Analysis. Rep. APEX-303, General Electric Co., Nov. 30, 1956.

9. Carver, J. G.: $\mathrm{U}^{235}$ Fission Gamma-Ray Source Data. Rep. XDC 60-11-63, General Electric Co., Oct. 5, 1960.

10. Draper, N. R.; and Smith, H.: Applied Regression Analysis. John Wiley \& Sons, Inc., 1966.

11. Lahti, Gerald P.: QADHD Point-Kernel Radiation Shielding Computer Code to Evaluate Propellant Heating and Dose to Crew During Engine Operation. NASA TM $\mathrm{X}-1397,1967$.

12. Evans, Robley D.: The Atomic Nucleus. McGraw-Hill Book Co., Inc., 1955. 
H. W.

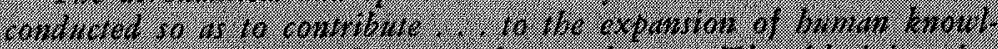

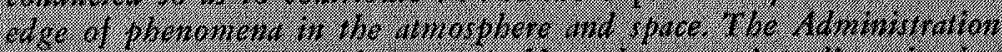

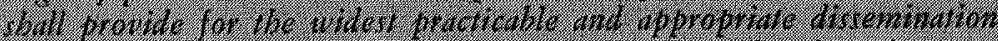

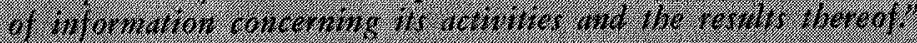

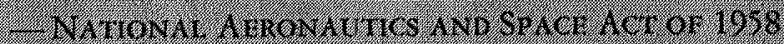

\section{NASA SCIENTIFIC AND TECHNICAT, PUBLICATIONS}

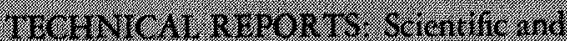

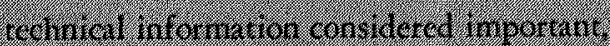

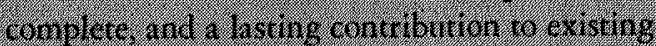
1.m.

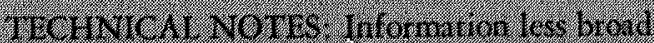
n.

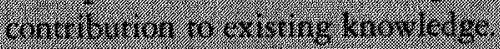

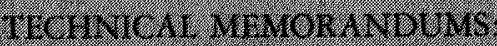

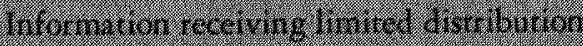
I.

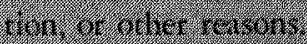

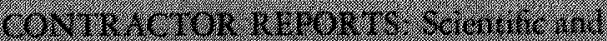

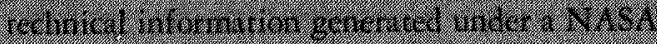

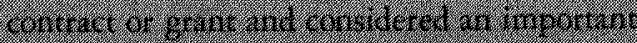

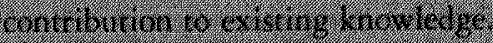

TECHNICAL TR ANSIATIONS: Intomation piblished in a fercetgn langunge considered to ment NASA distribution in Inglish.

SPLCIAI PUBLICATIONS: Intomation derived from or of thatue ro N/Xst activities. Pintications inclide conference procedelings:

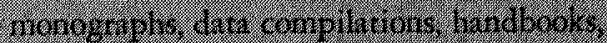

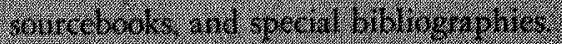

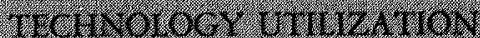

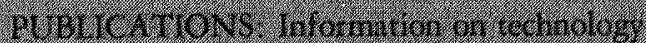

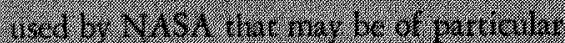

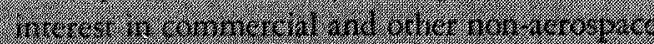

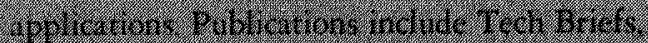

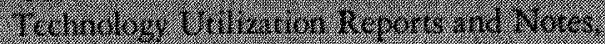

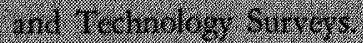

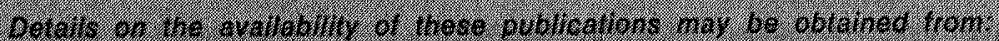

\section{SCIENTIEIC AND TECHMICAL INFORMATION DIVISION}

NATIONAL AERONAUTICS AND SPACE ADMINISTRATION W. Winton, D. C 20545 